\title{
Probleme des Bergsommertourismus
}

\section{Einleitung}

Im vorliegenden Artikel werden ausgewählte Probleme des Bergsommertourismus behandelt. Dabei sollen diejenigen Probleme zur Sprache kommen, die in der aktuellen Diskussion über die Krise im Alpentourismus eher etwas in den Hintergrund gedrängt werden. Es ist also nicht von der Konjunktur, den Wechselkursen oder der unzureichenden Gastfreundschaft die Rede, sondern von Aspekten wie der Aufenthaltsdauer, dem Image oder den touristischen Umsätzen.

Der alpine Sommertourismus hat eine lange Tradition und ist auch heute noch von großer Bedeutung. In der Literatur findet er allerdings keine seiner Bedeutung entsprechende Beachtung. Es gibt zwar viele Detailinformationen, kaum aber ein Werk, das sich explizit zum Bergsommertourismus äußern würde. Informationen müssen deshalb aus Abhandlungen zusammengetragen werden, die sich mit allgemeinen Fragen des Alpentourismus beschäftigen. Recht ergiebig ist dagegen eine Auswertung der Statistiken. Die Datenlage ist jedoch nur für die Hotellerie (Hotel- und Kurbetriebe) gut, für die Parahotellerie (Ferienwohnungen, Gruppenunterkünfte, Zelt- und Wohnwagenplätze und Jugendherbergen) ist sie lückenhaft. Dies ist bedauerlich, spielt doch die Parahotellerie in gewissen Gebieten eine sehr große Rolle. Ferner muß darauf hingewiesen werden, daß der Tagestourismus nur selten thematisiert wird. Das hat nichts mit einer Geringschätzung dieses Tourismussegmentes zu tun, sondern kann auf die unzureichende Datenlage (vgl. KASPAR/FREY 1993) zurückgeführt werden. Auch im vorliegenden Artikel gilt die Aufmerksamkeit vor allem dem Ferientourismus.

Der Artikel basiert auf einer Untersuchung über den Sommertourismus im Kanton Graubünden (NÖTHIGER 1996). Zusätzlich zur Auswertung der obengenannten Datenquellen wurde für diese Untersuchung eine Expertenbefragung bei lokalen Bündner Verkehrsvereinen und beim Verkehrsverein Graubünden durchgeführt. Der Region Graubünden wird daher besonderes Gewicht beigemessen. Nach Möglichkeit werden aber auch die übrigen Tourismusregionen der Schweiz (vgl. Abb. 1) mit einbezogen. Regionen, die keinen Anteil am Alpenraum haben (z. B. Zürich), sind nur für Vergleichszwecke von Interesse.

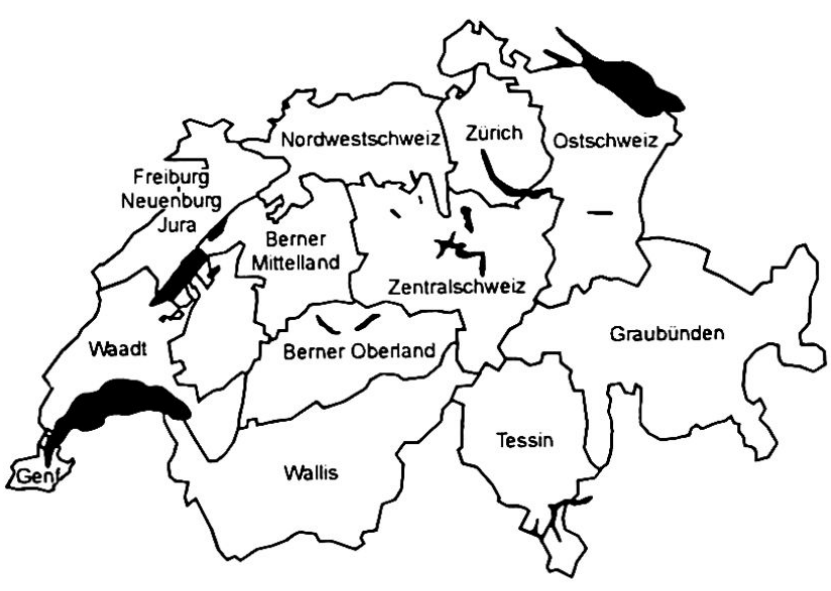

Abb. 1 Die zwölf Tourismusregionen der Schweiz (eigene Darstellung).

\section{Entwicklung des Bergsommertourismus}

Der alpine Sommertourismus kann auf eine lange Geschichte zurückblicken (vgl. z.B. SENGER 1962). Während langer Zeit, auch noch Jahrzehnte nachdem die ersten Wintergäste die Alpen entdeckten, war der Sommer die wichtigere Saison. Der langsame, aber kontinuierliche Bedeutungsverlust setzte erst in der Zwischenkriegszeit ein. Das heißt allerdings nicht, daß die Sommerlogiernächte (Mai bis Oktober) in der Folge stagnierten oder gar sanken. Abbildung 2 zeigt anhand der Hotelübernachtungen, welchen Aufschwung die Sommersaison in den 50er und 60er Jahren erlebte. Noch deutlicher war der Boom in der hier nicht dargestellten Parahotellerie. Die Übernachtungen im Winterhalbjahr nahmen indes noch weit stärker zu, und deshalb waren die Anteile der Sommersaison am Gesamtjahrestotal der Logiernächte seit Ende des Zweiten Weltkrieges fast ständig rückläufig.

Christian Nöthiger, dipl. Geograph, Kerenzerbergstraße 27, 8753 Mollis

Bruno Abegg, Dr., Geographisches Institut der Universität Zürich Irchel, Winterthurerstraße 190, 8057 Zürich 


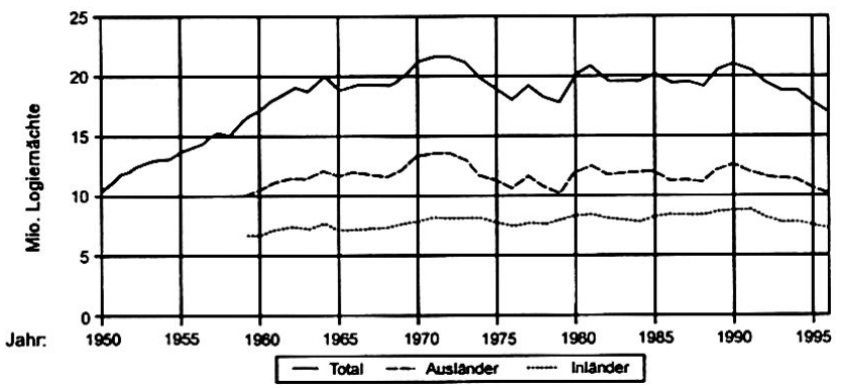

Abb. 2 Die Hotellogiernächte in der Schweiz in den Sommerhalbjahren 1950 bis 1996.

(Eigene Darstellung nach BUNDESAMT FÜR STATISTIK 1997 und früheren Jahrgängen.)

Für die Zeit von 1965 bis heute zeigt Abbildung 2 ein insgesamt stagnierendes Bild mit deutlichen Rückgängen in den 70er und 90er Jahren. Diese Einbrüche waren nicht sommerspezifisch, sondern betrafen auch die Wintersaison. Auffallend ist das Ausmaß des jüngsten Logiernächterückgangs: im Sommer 1996 lagen die Logiernächte in der Schweizer Hotellerie mit 16,8 Mio. Übernachtungen wieder etwa im Bereich des Ergebnisses von 1960.

Im Zuge des Nachfrageaufschwungs seit Kriegsende wurde auch das touristische Angebot ausgebaut. Ein Großteil des Wanderwegnetzes entstand, und auch die Zahl der touristischen Transportanlagen (Sesselbahnen, Skilifte, Kabinenbahnen) nahm rapide zu. Die Transportanlagen wurden zwar vor allem im Hinblick auf die Wintersaison ausgebaut, ein Teil der bodenunabhängigen Anlagen bereicherte aber auch das Sommerangebot. Gleichzeitig wurde das Sportangebot ausgedehnt. Sommersportarten, die bereits in der zweiten Hälfte des letzten Jahrhunderts verbreitet waren (wie z. B. Tennis, Golf und Bergsteigen), wurden durch neue Angebote ergänzt. Die größte Ausdehnung erfuhr das Sportangebot jedoch in den letzten zwanzig Jahren. In diesen Zeitraum fällt z.B. das Aufkommen von Delta- und Gleitschirmfliegen, Mountainbiking oder Rafting (vgl. LORCH 1995). $\mathrm{Zu}$ den Verlierern der Nachkriegszeit gehörten der Luftkurtourismus und zunächst auch der Bädertourismus. Die Heilbäder, welche die Bäderkrise überstanden haben, zählen heute aber wieder zu den relativ krisensicheren Angeboten. Durch die Einführung des Ganzjahresbetriebs, umfangreiche Sanierungen und durch das Ansprechen neuer Gästesegmente ist es gelungen, verlorenes Terrain wieder wettzumachen.

Abbildung 3 zeigt die aktuelle Verteilung der Sommerlogiernächte auf die einzelnen Regionen der Schweiz. Spitzenreiter ist der Kanton Graubünden mit einem Anteil von knapp 15\%. Dieser Anteil hat sich aufgrund der vergleichsweise positiven Entwicklung in der Zeit von 1984 bis 1994 um drei Prozentpunkte erhöht. Noch größer ist die Dominanz von Graubünden allerdings im Winter. So betrug der Bündner Anteil an den schweizerischen Hotellogiernächten im Winterhalbjahr 1995/96



Abb. 3 Anteile der Regionen an den Logiernächten der Hotel- und Kurbetriebe in der Schweiz im Sommerhalbjahr 1996. (Eigene Berechnungen nach BUNDESAMT FÜR STATISTIK 1997.)

$\mathrm{GR}=$ Graubünden $/ \mathrm{ZS}=$ Zentralschweiz $/ \mathrm{BO}=$ Berner Oberland / TI = Tessin / VS = Wallis / ZH = Zürich / VD = Waadt / $\mathrm{GE}=$ Genf / OS = Ostschweiz / NWS = Nordwestschweiz / $\mathrm{BM}=$ Berner Mittelland / FNJ = Freiburg-Neuenburg-Jura

23,6\% (BUNDESAMT FÜR STATISTIK 1996b). Graubünden und das Wallis sind die beiden einzigen Regionen, in denen mehr als die Hälfte der gesamten Hotellogiernächte im Winter gezählt werden. In allen anderen Regionen - so z. B. auch im Berner Oberland - überwiegt der Sommeranteil (vgl. Abb. 4). Die geläufige These, eine dominierende Wintersaison sei typisch für das schweizerische Berggebiet, ist aufgrund der saisonalen Verteilung der Hotellogiernächte also nicht zulässig. Gesamtschweizerisch machen die Sommerlogiernächte trotz des langjährigen Bedeutungsverlustes der Sommersaison immer noch $56 \%$ der Jahresübernachtungen aus.

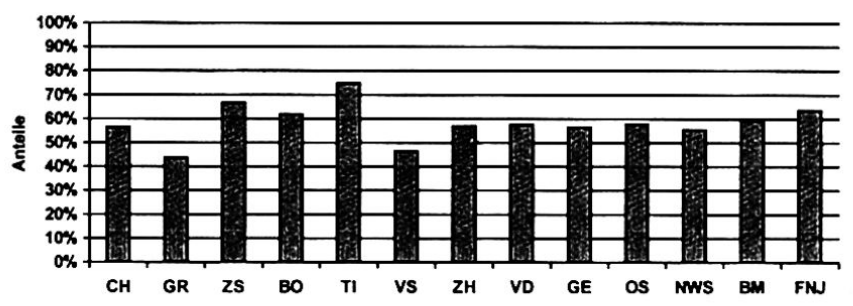

Abb. 4 Anteil des Sommerhalbjahres an den Gesamtlogiernächten in Hotel- und Kurbetrieben des Jahres 1995/96. (Eigene Berechnungen nach BUNDESAMT FÜR STATISTIK 1996b und 1997.)

$\mathrm{CH}=$ Gesamtschweiz / GR = Graubünden / ZS = Zentralschweiz / BO = Berner Oberland / TI = Tessin / VS = Wallis / $\mathrm{ZH}=$ Zürich / VD = Waadt / GE = Genf / OS = Ostschweiz / NWS $=$ Nordwestschweiz $/ \mathrm{BM}=$ Berner Mittelland $/ \mathrm{FNJ}=$ Freiburg-Neuenburg-Jura

Die obigen Ausführungen haben gezeigt, daß das Sommerhalbjahr auch über ein Jahrhundert nach der Einführung des Wintersports nicht zur touristischen Nebensaison verkommen ist. Seine Bedeutung rechtfertigt also durchaus eine etwas genauere Betrachtung seiner spezifischen Merkmale und Probleme. 


\section{Probleme des alpinen Bergsommertourismus}

Das größte aktuelle Problem der Schweizer Tourismusbranche ist mit Sicherheit der seit einigen Jahren zu verzeichnende Logiernächterückgang. Von dieser Entwicklung sind Sommer- und Wintersaison gleichermaßen betroffen, wobei vor allem die ungünstigen Wechselkurse, die schlechte Konjunkturlage und die Globalisierung der Tourismusmärkte für die derzeitige Misere im Schweizer Tourismus verantwortlich gemacht werden. Neben diesen Faktoren gibt es aber auch noch andere, die eine Rolle spielen, und diese sollen hier - sofern sie eine sommerspezifische Ausprägung haben - thematisiert werden. Es handelt sich dabei um Aspekte wie die Aufenthaltsdauer, die touristischen Umsätze, die Bergbahnfrequenzen, das Image und die Konkurrenzsituation. Für manch einen Tourismusanbieter dürften diese Aspekte zurzeit nicht im Zentrum stehen, sie müssen aber bei einer Diskussion der aktuellen Situation gleichwohl berücksichtigt werden. Weiter werden die besagten Aspekte auch dann noch von Bedeutung sein, wenn sich die allgemeinen Rahmenbedingungen (Konjunktur, Wechselkurse) für den alpinen Sommertourismus wieder verbessern werden.

\subsection{Die Aufenthaltsdauer und-häufigkeit}

Für die Tourismusbranche ist nicht nur entscheidend, wie viele Gäste kommen, sondern auch, wie lange diese bleiben. Bei der durchschnittlichen Aufenthaltsdauer läßt sich seit Ende des Zweiten Weltkrieges ein abnehmender Trend feststellen. Deutlich sichtbar wird dies am Beispiel des Bündner Sommertourismus (Abb. 5). 1946 verbrachten die Sommergäste durchschnittlich 10,8 Tage in den Hotel- und Kurbetrieben Graubündens; 1996 waren es noch 3,3 Tage. Ebenfalls abnehmend, aber insgesamt immer etwas höher war die Aufenthaltsdauer in der Parahotellerie. 1965 betrug sie im Bündnerland 9,3 Tage; 1990 waren es noch 5,9 Tage.

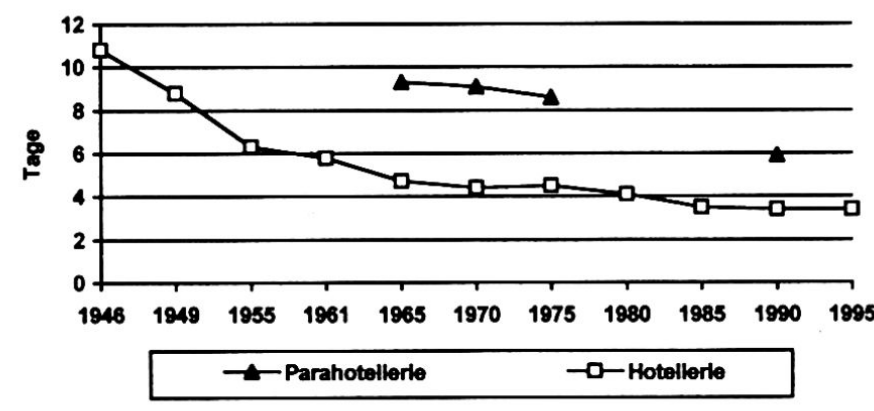

Abb. 5 Durchschnittliche Aufenthaltsdauer im Kanton Graubünden in den Sommerhalbjahren 1946 bis 1995. (Eigene Berechnungen nach BUNDESAMT FÜR STATISTIK 1997 und früheren Jahrgängen.)
Die Aufenthaltsdauer ging nicht nur in der Sommersaison, sondern auch im Winterhalbjahr zurück. Ein typisches Merkmal des Bergtourismus besteht nun aber darin, daß die Sommeraufenthalte in der Regel kürzer sind (vgl. Abb. 6). Im Tourismusjahr 1995/96 wiesen z. B. nur das Tessin und das Berner Mittelland eine (geringfügig) längere Aufenthaltsdauer im Sommer auf; in allen anderen Regionen war die Aufenthaltsdauer im Winter höher. Besonders deutlich ist der saisonale Unterschied in Graubünden, im Wallis und im Berner Oberland. Man kann daher annehmen, daß dieser Unterschied gerade für das Berggebiet typisch ist. Erklären läßt sich dies mit den im Sommer beliebteren Kurzferien (z. B. Wochenende) und dem größeren Durchreiseverkehr ans Mittelmeer. Im Winter sind dagegen die klassischen ein- bis zweiwöchigen Skiferien nach wie vor weit verbreitet.



Abb. 6 Durchschnittliche Aufenthaltsdauer in Hotel- und Kurbetrieben 1995/96 in der Schweiz und ihren Regionen. (Eigene Berechnungen nach BUNDESAMT FÜR STATISTIK 1996b und 1997.)

$\mathrm{CH}=$ Gesamtschweiz / GR = Graubünden / ZS = Zentralschweiz / BO = Berner Oberland / TI = Tessin / VS = Wallis / $\mathrm{ZH}=$ Zürich / VD = Waadt / GE = Genf / OS = Ostschweiz / NWS $=$ Nordwestschweiz $/ \mathrm{BM}=$ Berner Mittelland $/ \mathrm{FNJ}=$ Freiburg-Neuenburg-Jura

Neben der Aufenthaltsdauer unterscheidet sich auch die Aufenthaltshäufigkeit. Im Sommer ist der Anteil der Feriengäste, die die Schweiz zum erstenmal besuchen, fast überall größer als im Winter. Dafür ist der Anteil der Stammgäste kleiner: im Sommer 1988 betrug der Anteil der Gäste, die sich mindestens zum viertenmal in ihrer jeweiligen Ferienregion aufhielten, $35 \%$; im Winter 1988/89 dagegen 43\% (KÜNZI/SCHMIDHAUSER 1989b, S. 76). In den Bergregionen sind die Stammgastanteile besonders hoch; die saisonalen Unterschiede bestehen aber genauso. In Graubünden machten die Stammgäste im Winter $76 \%$ und im Sommer $51 \%$ aus. Eine kürzere durchschnittliche Aufenthaltsdauer und ein kleinerer Anteil von Stammgästen stellen das Tourismusmarketing vor große Herausforderungen. Um die gleiche Zahl von Übernachtungen wie im Winter zu erzielen, muß im Sommer eine größere Zahl von Gästen 
angeworben werden. Um die Übernachtungszahl von zwei durchschnittlichen Wintergästen zu erreichen, müssen z.B. in Graubünden etwa drei Sommergäste zu einem Hotelaufenthalt animiert werden. Ist einer der beiden Wintergäste zudem Stammgast (und muß daher nicht mehr neu gewonnen werden), vergrößert sich der saisonale Unterschied im Werbeaufwand weiter. Neben dem Anwerben von neuen Gästen kann auch versucht werden, die Aufenthaltsdauer von bereits anwesenden Gästen (z.B. von Durchreisenden) zu erhöhen. Beide Ziele verfolgte der Verkehrsverein Graubünden, als er im Sommer 1996 mit einem Handzettel auf die Attraktionen im näheren Umkreis der San-Bernardino-Route hinwies (unter dem Titel «Entlang der A13 - Wir wollen Sie vom rechten Weg abbringen»).

\subsection{Die direkten touristischen Umsätze}

Von den touristischen Übernachtungen profitieren nicht nur die Beherbergungsbetriebe. Zu den Nutznießern gehören auch das Gastgewerbe, die Bergbahnen, der öffentliche Verkehr, die Lebensmittelläden, Banken, Tankstellen, Sportgeschäfte sowie deren Zulieferer. Die Höhe der Ausgaben der Feriengäste ist daher für ein Tourismusgebiet von vitalem Interesse. Man bezeichnet diese Ausgaben auch als direkte touristische Umsätze (wobei mit indirekten touristischen Umsätzen Zahlungen an Zulieferer wie z. B. Landwirte oder Baufirmen bezeichnet werden).
In der letzten großangelegten Gästebefragung der Schweiz (sog. TOMAS-Umfrage $=$ Touristisches Marktforschungssystem Schweiz) von 1988/89 versuchte man, die Höhe der durchschnittlichen Tagesausgaben zu eruieren (KÜNZI/SCHMIDHAUSER 1989 a, S. $67 \mathrm{f}$., und 1989 b, S. 71 f.). Es wurde nach dem Total der Unterkunfts-, Getränke- und Verpflegungskosten sowie nach den übrigen Kosten gefragt. Zu den übrigen Kosten zählen die Ausgaben für Sport, Unterhaltung, Ausflüge, Einkäufe, Kongreßkosten und Nebenauslagen. Abbildung 7 zeigt die Antworten auf diese Frage nach Regionen und Saison aufgeschlüsselt. Bezüglich des saisonalen Unterschiedes ergibt sich kein einheitliches Bild, und es ist an dieser Stelle auch nicht möglich, auf die Situation jeder einzelnen Region einzugehen. Am Beispiel von Graubünden erkennt man indes, daß die Ausgaben im Sommerhalbjahr generell tiefer sind als im Winterhalbjahr. Dies dürfte für Bergregionen mit stark ausgeprägtem Skitourismus typisch sein.

Eine Wertschöpfungsstudie in der Region Mittelbünden (ZEGG/MATTER/INÄBNIT 1993) bestätigte das Ergebnis, welches sich aus der TOMAS-Umfrage für ganz Graubünden ergeben hatte. Wie aus Abbildung 8 zu entnehmen ist, gaben die Feriengäste, egal, in welchen Unterkunftskategorien sie untergebracht waren, im Winter mehr aus als im Sommer. Dasselbe gilt in besonderem Maße auch für die in derTOMAS-Umfrage nicht berücksichtigten Tagesgäste.

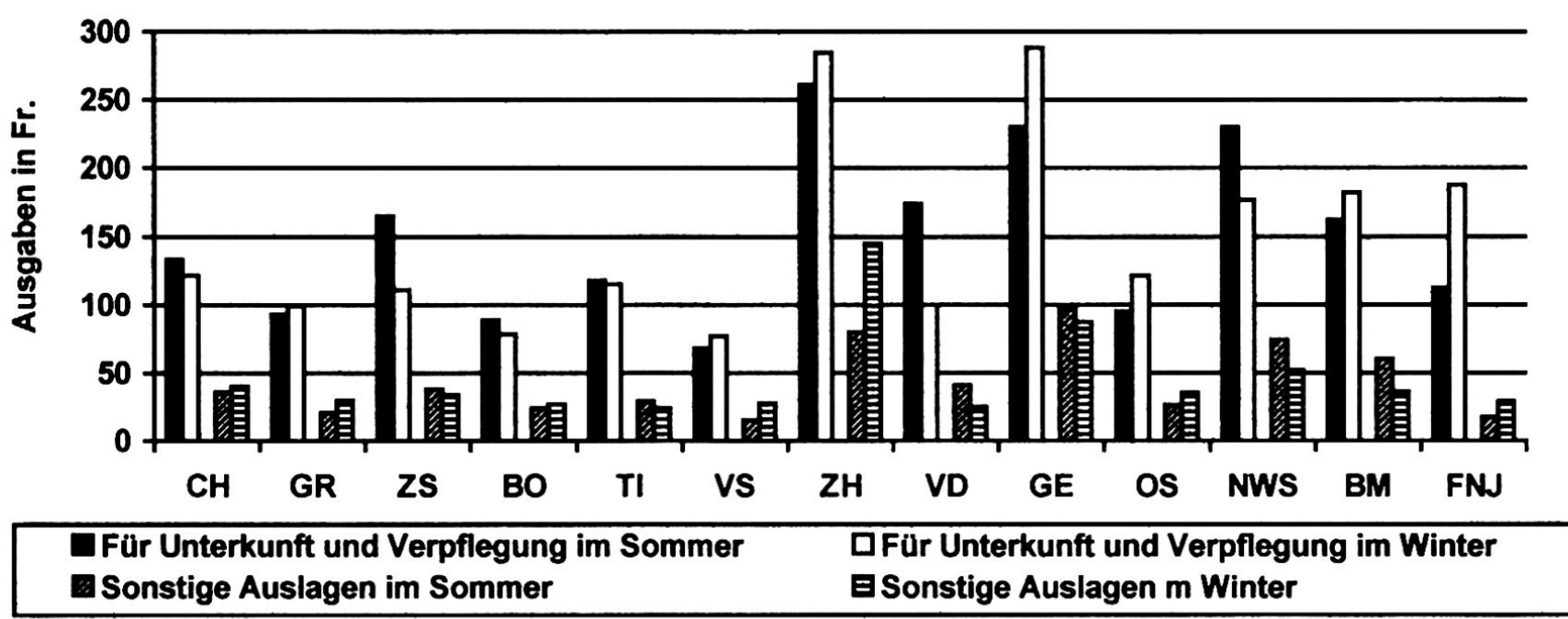

Abb. 7 Durchschnittliche Tagesausgaben eines Ferienaufenthalters (1988/89).

(Eigene Darstellung nach KÜNZI/SCHMIDHAUSER 1989a, S. 68 und 1989b, S. 72.)

$\mathrm{CH}=$ Gesamtschweiz $/ \mathrm{GR}=$ Graubünden $/ \mathrm{ZS}=$ Zentralschweiz $/ \mathrm{BO}=$ Berner Oberland $/ \mathrm{TI}=$ Tessin $/ \mathrm{VS}=\mathrm{Wallis} / \mathrm{ZH}=$ Zürich / VD = Waadt / GE = Genf / OS = Ostschweiz / NWS = Nordwestschweiz / BM = Berner Mittelland / FNJ = FreiburgNeuenburg-Jura 


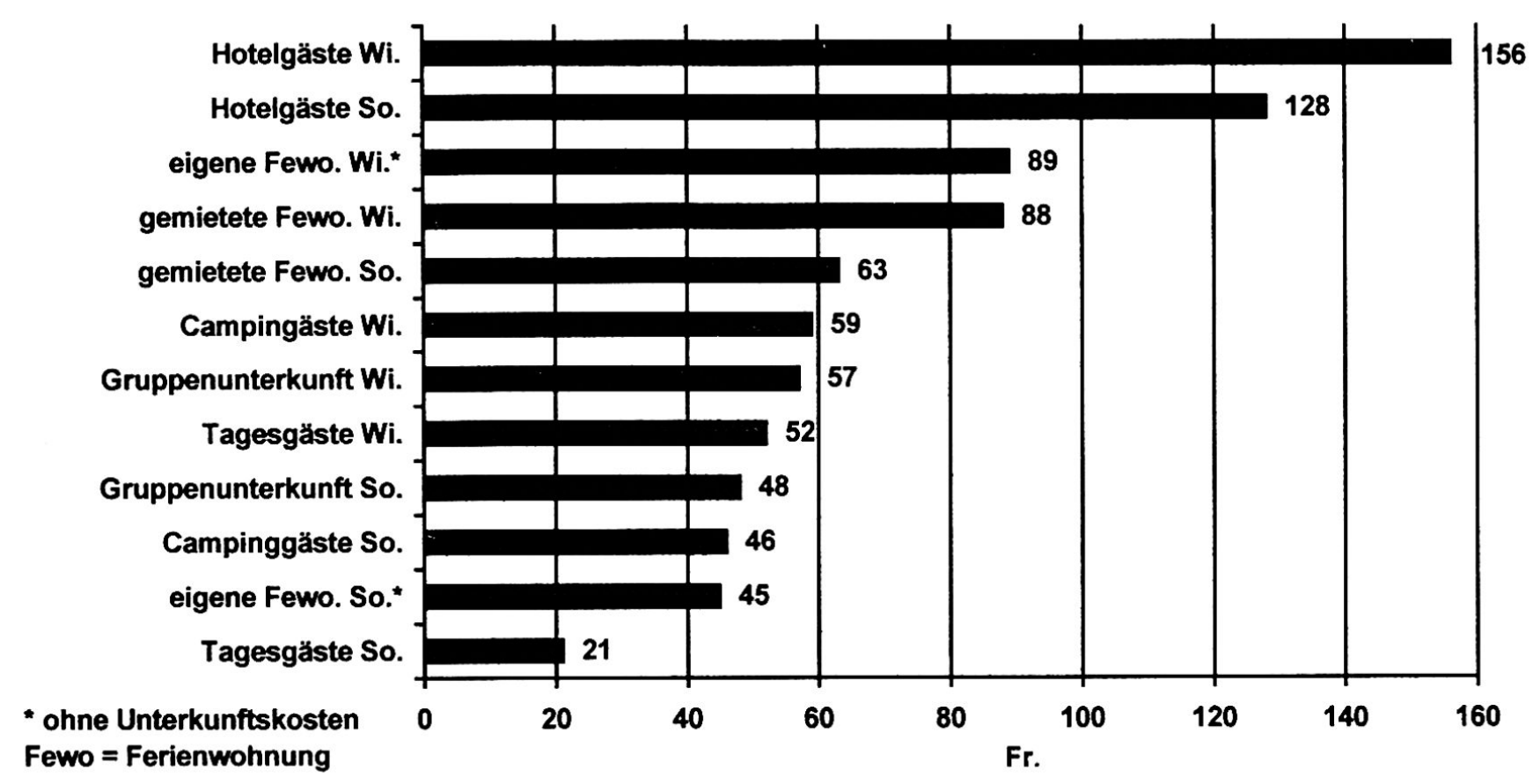

Abb. 8 Rangliste der durchschnittlichen Tagesausgaben für die einzelnen Gästekategorien im Winter und Sommer 1991/1992 in der Region Mittelbünden.

(ZEGG/MATTER/INÄBNIT 1993, S. 13)

Dieser saisonale Unterschied hat zur Folge, daß die finanzielle Bedeutung der Sommersaison wesentlich geringer ist, als es aufgrund der Logiernächtezahl zu erwarten wäre. So bringen die Sommergäste dem Kanton Graubünden zwar etwa $42 \%$ der Übernachtungen, aber nur ungefähr $25-30 \%$ des direkten touristischen Jahresumsatzes (HARTMANN 1995, S. 48). Für diesen Umstand lassen sich folgende Gründe anführen: Bei den Unterkunftskosten können saisonale Unterschiede auftreten, wenn die Hoteliers im Sommer mit tieferen Gewinnmargen operieren, um eine genügend große Auslastung zu erreichen. So gibt es verschiedene Hotels, die im Sommer mit Busreisegruppen (z. B. Werbefahrten) arbeiten, die pro Kopf weniger Ertrag bringen als Individualreisende. Dieser Strategie sind jedoch durch die Fixkosten relativ enge Grenzen gesetzt.

Die TOMAS-Umfrage hat auch ergeben, daß die Bündner Wintergäste öfters ein Restaurant besuchen als die Sommergäste. Dies ist nicht weiter erstaunlich, lädt doch das Sommerklima eher zum Picknicken ein. Da Picknicken günstiger ist als der Besuch eines Restaurants, schlägt sich das in den Ausgaben für «Unterkunft und Verpflegung» nieder.

Bei den sonstigen Ausgaben dürften vor allem die Kosten im Bereich «Sport» zu saisonalen Unterschieden führen. Die winterlichen Massensportarten (Skifahren, Snowboarden, Langlauf) bringen den Sportartikelge- schäften wesentlich mehr Umsatz als das Wandern. Und das Wandern nimmt in der Beliebtheitsskala der Sommergäste nach wie vor den ersten Rang ein und konnte bisher nicht von neueren, «materialintensiveren» Sommersportarten (wie Mountainbiking oder Gleitschirmfliegen) überflügelt werden. Noch stärker als die saisonal unterschiedlichen Ausgaben in den Sportgeschäften fallen aber die Unterschiede bei den Ausgaben für die Bergbahnen ins Gewicht.

\subsection{Die Bergbahnfrequenzen}

Die ersten Bergbahnen wurden in der Schweiz bereits Ende des letzten Jahrhunderts gebaut und dienten vor allem der Erschließung von Aussichtsbergen für die Sommergäste (vgl. RÖTHLISBERGER 1959). Die nach dem Zweiten Weltkrieg entstandenen Sessel-, Kabinen- und Gondelbahnen waren dagegen in erster Linie für eine Nutzung durch den Wintersport vorgesehen. Es erstaunt deshalb nicht, daß sich nur ein Teil dieser Anlagen für den Sommerbetrieb eignet. Eine Eignung für den Sommerbetrieb bedeutet aber noch nicht, daß sich der Sommerbetrieb auch lohnt. Im Gegenteil, zahlreiche Bergbahngesellschaften stehen vor dem Problem, daß die Erträge aus dem Sommergeschäft die Betriebskosten nicht aufwiegen. 


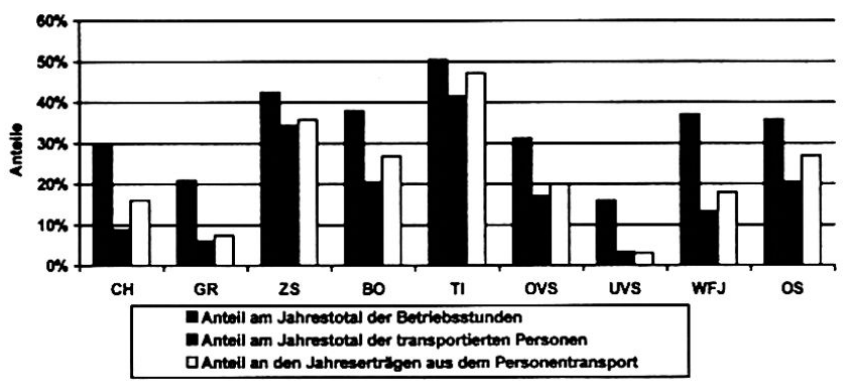

Abb. 9 Anteile des Sommerhalbjahres an den Gesamtjahresergebnissen 1994 der Bergbahngesellschaften der Schweiz (Sessellifte, Kabinen- und Gondelbahnen). (Eigene Berechnungen nach BUNDESAMT FÜR STATISTIK 1996a.)

$\mathrm{CH}=$ Gesamtschweiz / GR = Graubünden / ZS = Zentralschweiz $/ \mathrm{BO}=$ Berner Oberland $/ \mathrm{TI}=$ Tessin / OVS = Ober wallis / UVS = Unter- und Zentralwallis / WFJ = Waadt-Freiburg-Jura / OS = Ostschweiz

Abbildung 9 zeigt, daß in allen Regionen der Schweiz die Anteile des Sommers an den transportierten Personen des Gesamtjahres tiefer sind als die Anteile an den Betriebsstunden. Im Oberwallis wurden z. B. in $31 \%$ der Jahresbetriebszeit nur $17 \%$ der Jahrespassagiere transportiert; im Unter- und Zentralwallis in 16\% der Jahresbetriebszeit nur gerade 3\% der Jahrespassagiere. Dementsprechend tief sind auch die Sommeranteile an den Jahreserträgen aus dem Personentransport. Sie sind jedoch meist höher als die Anteile an den Passagieren, was darauf hinweist, daß eine durchschnittliche Bergbahnfahrt im Sommer teurer zu stehen kommt als im Winter (wegen Tages- und Saisonkarten u. ä.).

Aus Abbildung 9 wird deutlich, da $\beta$ das Sommergeschäft für die meisten Bergbahnunternehmen keine Goldgrube darstellt. Man muß weiter bedenken, daß hier Durchschnittswerte wiedergegeben sind. Natürlich gibt es einige Ausflugsbahnen, die im Sommer ausgezeichnet arbeiten (Beispiel Titlisbahnen), andere aber, wie

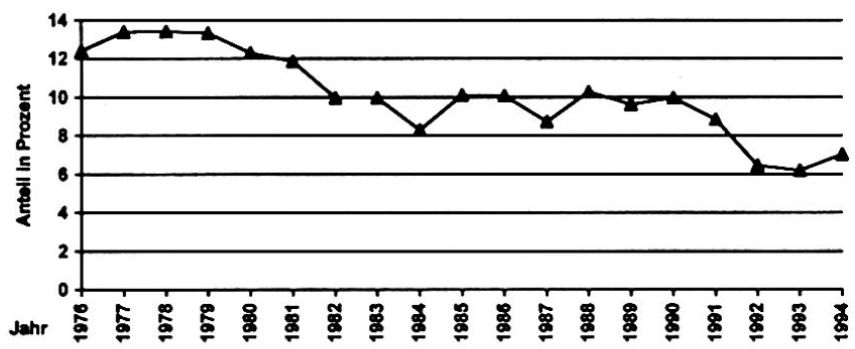

Abb. 10 Anteil der in den Sommerhalbjahren 1976 bis 1994 beförderten Personen an den Gesamtjahrestotalen aller Bergbahnen Graubündens.

(Eigene Berechnungen nach BUNDESAMT FÜR STATISTIK 1996a und früheren Jahrgängen.) gewisse Bergbahngesellschaften Graubündens, erwirtschaften weniger als $1 \%$ ihrer Jahreserträge im Sommer. Zudem gab es in den letzten 20 Jahren einen Trend zu abnehmenden Sommeranteilen am Personentransport (vgl. Abb. 10). Dieser läßt sich nicht alleine durch den Neubau von ausschließlich im Winter betriebenen Bahnen erklären.

Der Grund für die tiefen Anteile der Sommersaison liegt auf der Hand. Skifahrer benützen die Bergbahnen mehrmals täglich als Aufstiegshilfe. Ein Wanderer oder ein Ausflugstourist kommt dagegen im besten Fall auf eine Berg- und eine Talfahrt pro Tag. Die Frage ist, was angesichts dieser Situation getan werden kann. Aufgrund wirtschaftlicher Überlegungen müßten viele Bergbahngesellschaften den Sommerbetrieb eigentlich einstellen. Solche Absichten werden aber von den übrigen Tourismusanbietern nicht goutiert. Besitzer von Unterkünften und Gaststätten sowie Verkehrsvereine befürchten, daß eine Aufgabe des sommerlichen Bergbahnbetriebs zu einem Attraktivitätsverlust des Ferienortes führen könnte. Sie fordern deshalb von den Bergbahngesellschaften dessen Aufrechterhaltung. Zu einer Mitfinanzierung des Sommerbetriebs sind sie aber normalerweise nicht in der Lage (oder nicht gewillt). Viele Bergbahnbetreiber müssen deshalb die Verluste aus dem Sommergeschäft mit Gewinnen aus dem Winter kompensieren. Einige versuchen, die Sommerfrequenzen durch spezielle Angebote bei den Bergstationen (Sommerrodelbahnen, Kunstausstellungen) oder durch das Ansprechen neuer Kundensegmente (Mountainbiker, Gleitschirmflieger) zu erhöhen. Eine Annäherung der Sommer- an die Winterfrequenzen läßt sich aber auch auf diesem Weg kaum erreichen.

\subsection{Das Image}

Beim Entscheid fur ein bestimmtes Ferienziel spielen die Vorstellungen und Erwartungen der potentiellen Touristen eine große Rolle. Ein gutes Image ist deshalb für die Tourismusbranche äußerst wichtig. Wie eine Studie im Auftrag von "Schweiz Tourismus» (VERKEHRSVEREIN GRAUBÜNDEN 1994, S. 33-35) ergeben hat, ist das Image von Bergsommerferien in der Schweiz aber eher problematisch. Es herrscht die weitverbreitete Meinung, Bergsommerferien seien vor allem etwas für ältere Leute, das Angebot bestünde nur aus Wandern und man könne nichts erleben. Weiter haben Sommerferien in den Bergen einen relativ geringen Prestigewert. Winterferien in den Bergen werden dagegen mit einem großen Angebot, mit «Lifestyle» und «Jet Set» verbunden und haben ein hohes Prestige.

Der Eindruck eines höheren Durchschnittsalters im Sommer wird durch die TOMAS-Umfrage bestätigt (KÜNZI/SCHMIDHAUSER 1989b, S. 84). Die Sommergäste waren im Schnitt 39 Jahre alt, und der Anteil der über 60 jährigen betrug $21 \%$, während die Wintergäste durchschnittlich 35jährig waren und Personen über $6013 \%$ ausmachten. Das Problem besteht nun anscheinend dar- 
in, daß mit dem größeren Anteil älterer Gäste «Langeweile» und ein eingeschränktes Angebot verbunden wird. $\mathrm{Da} \beta$ man im Sommer «nur» wandern könne, ist indes ein Eindruck, der allenfalls in den 50er und 60er Jahren noch zutraf. Heute sind die Möglichkeiten z.B. zur sportlichen Betätigung bedeutend größer als damals (und wahrscheinlich sogar vielfältiger als im Winter). $\mathrm{Da}$ sich dies noch nicht genügend herumgesprochen hat, deutet einerseits auf die Langlebigkeit von Images hin. Auf der anderen Seite wird aber auch ein gewisser Handlungsbedarf im Tourismusmarketing sichtbar. Das hat man auch in der Tourismusbranche realisiert, und man versucht, das Angebot mit Trendsportarten zu erweitern und neue Wege in der Vermarktung einzuschlagen.

\subsection{Die Konkurrenzsituation}

Für Skiferien sind die Alpen nach wie vor erste Adresse in Europa. Wer sich im Sommer aber beispielsweise für Tennis- oder Golfferien interessiert, hat eine Vielzahl von möglichen Feriengebieten zur Auswahl. Dieses Beispiel soll veranschaulichen, daß die Konkurrenz für die Ferienanbieter des Alpenraumes im Sommer wesentlich größer ist als im Winter. Im Winter konkurrenzieren sich vor allem die verschiedenen Alpenregionen. Im Sommer treten daneben eine Vielzahl von weiteren Regionen zwischen Nordkap und Mittelmeer sowie in zunehmendem Maße Gebiete in Übersee als Konkurrenten auf. Die Globalisierung der Tourismusmärkte hat dazu geführt, daß Distanzen und Reisezeiten eine wesentlich geringere Rolle spielen als noch vor zehn Jahren und daß sich die Konkurrenzsituation weiter verschärft. Zwar hat sich auch die Zahl der Anbieter (z. B. von Badeferien) in der Wintersaison vergrößert, aber die Angebotsunterschiede zwischen dem Alpenraum und seinen Konkurrenten in Übersee sind im Winter entschieden größer als im Sommer.

Um die Position des Schweizer Bergsommertourismus im internationalen Wettbewerb bestimmen zu können, sind einige Ausführungen zu seinen Stärken und Schwächen nötig. Als Grundlage können Umfragen in der Branche (z. B. BALDENWEG-BÖLLE 1996) oder unter potentiellen Gästen (z. B. NÜTZı 1997) dienen. Deren Resultate sind zumeist ähnlich. Als Stärken der Schweiz kann man die vergleichsweise intakte Natur, die zuverlässige Basisinfrastruktur (z. B. öffentlicher Verkehr), die Sicherheit, die Sauberkeit und die vielfältigen Möglichkeiten (z. B. im Sport) auf kleinem Raum bezeichnen. Dies gilt im Sommer wie im Winter. Zu den größten Schwächen gehören mit Sicherheit das Preisniveau bzw. das Preis-Leistungs-Verhältnis und die z.T. mangelhafte Gastfreundschaft und Dienstleistungsbereitschaft. Diese sind ebenfalls saisonunabhängig und werden deshalb an dieser Stelle nicht ausführlich besprochen. Zwei Schwächen sind jedoch sommerspezifisch. Die eine ist das Image, auf das bereits im vorangehenden Abschnitt eingegangen wurde, und die andere das Wetter.
Im Winter treten häufig Hochdrucklagen auf mit herrlichem Wetter in den Bergen und Nebel im Flachland. Im Sommer sind die Unterschiede zwischen Gebirgsund Flachlandklima geringer, und längere Regenperioden sind da wie dort keine Seltenheit (GENSLER 1979, S. 138). Das Sommerklima in den Alpen wird deshalb von den Feriengästen als weniger attraktiv wahrgenommen als das Winterklima (KÜNZI/SCHMIDHAUSER 1989a, S. 47, und 1989 b, S. 49). Dies ist für den Bergsommertourismus ungünstig, weil ein Großteil der Konkurrenz (z. B. das Mittelmeer) im Sommerhalbjahr sonnensicher ist. Der Einfluß des Wetters wird um so größer, je kurzfristiger die Ferien gebucht werden. Wer ein halbes Jahr im voraus bucht, überlegt sich kaum, wie das Wetter zum Zeitpunkt der Reise sein wird. Dauerregen in der Woche vor Ferienbeginn lockt Touristen, die sich kurzfristig entscheiden («Last-Minute»), aber kaum in den Alpenraum. Gerade die Sommergäste gaben bereits in der TOMAS-Umfrage aus dem Jahre 1988 an, kurzfristiger zu buchen als die Wintergäste (KÜNZI/SCHMIDHAUSER 1989b, S. 76), und der Trend zum spontanen Ferienentscheid hat sich in den letzten Jahren noch verstärkt. Das bedeutet, daß der Bergsommertourismus in zunehmendem Maße vom Wetter abhängig wird, das sowieso schon einer seiner Schwachpunkte ist. Auch wenn sich die allgemeinen Rahmenbedingungen für den Schweizer Tourismus wieder verbessern sollten, kann also ein verregneter Sommer der Branche immer noch einen dicken Strich durch die Rechnung machen.

Die Möglichkeiten, etwas dagegen zu unternehmen, sind beschränkt. Mit wetterunabhängigen Angeboten lassen sich zwar ein paar Regentage überbrücken; sie sind jedoch kaum ausschlaggebend für den Aufenthalt in einer bestimmten Bergregion. Eine Ausnahme bilden die Heilbäder und Kurbetriebe. Übers Ganze gesehen befriedigen diese aber nur die Nachfrage einer Marktnische und sind keine echte Alternative für das Gros der Ferienorte. Ein gewisses Potential für jene bestünde allenfalls noch in Frühbuchungsrabatten. Ansonsten muß der Bergsommertourismus mit dem Wettbewerbsnachteil des unsicheren Wetters leben.

Das bedeutet, daß die Schweizer Tourismusbranche um so stärker an ihren übrigen Schwächen arbeiten muß. Es reicht im heutigen Umfeld nicht mehr, sich nur auf die traditionellen Stärken zu verlassen. Dies namentlich deshalb, weil die meisten dieser Stärken für einen großen Teil der potentiellen Gäste nicht den gleichen Stellenwert besitzen wie die Bereiche, wo die Schweiz nicht besonders wettbewerbsfähig ist (Preis-Leistungs-Verhältnis, Gastfreundschaft, Wetter). Daß man das offenbar zu lange nicht gemerkt hat, ist mit ein Grund, weshalb sich die Schweiz in der heutigen Konkurrenzsituation eher schwertut und im internationalen Tourismusgeschäft nicht mehr zu den Gewinnern gehört. 


\section{Ausblick}

Wenn man den gängigen Tourismusprognosen Glauben schenken darf, so hat der Bergsommertourismus eigentlich gute Aussichten. Die meisten Autoren (z.B. MÜLLER/KASPAR/SCHMIDHAUSER 1991) sind sich einig, daß Bergferien im Sommer ein vergleichsweise hohes Entwicklungspotential haben. Läuft es dem ins Stocken gekommenen Schweizer Tourismus wieder besser, dürfte also auch der Bergsommertourismus wieder in Fahrt kommen. Wie gezeigt wurde, ist der Bergsommertourismus aber mit diversen Problemen konfrontiert, die nicht einfach der aktuellen Krise zugeschrieben werden können, sondern auch anderen Ursprungs sind. Das bedeutet, daß selbst bei einer Verbesserung der Rahmenbedingungen (konjunktureller Aufschwung in den wichtigsten Herkunftsländern der Gäste, Verbesserung der Währungsrelationen usw.) und einem Abbau der notorischen Schwächen (Preis-Leistungs-Verhältnis, Dienstleistungsbereitschaft usw.) Handlungsbedarf besteht. Der Bergsommertourismus ist ein «reifes» Tourismusprodukt - er ist gewissermaßen in die Jahre gekommen und bedarf einer umfassenden Modernisierung. Schweiz Tourismus setzt weiterhin auf die Karte «Bergsommer» mit seiner klassischen Ausprägung, dem Wandern. Dies ist sinnvoll, gilt es doch bestehende Stärken zu erhalten. Daneben muß aber das Angebot dynamisiert und zeitgemäßer ausgestaltet werden (HARTMANN 1997). Diese Dynamisierung soll dazu führen, daß das traditionelle Kerngeschäft wiederbelebt und neue Gästesegmente dazugewonnen werden können. Im Vordergrund stehen dabei Trendsportarten im weitesten Sinne sowie Angebote im Bereich «Wellness». All diese Anstrengungen müssen von einer Imagekorrektur begleitet werden.

Was allerdings auch in Zukunft Sorgen bereiten dürfte, ist die sinkende Aufenthaltsdauer. Allen Tourismusprognosen gemein ist die Aussage, daß der Trend zu Kurzferien anhalten wird. Laufen die Alpen Gefahr, eine Kurzurlaubsdestination oder gar ein Tagesausflugsgebiet zu werden? Diese Befürchtung ist in ihrer Tendenz sicher nicht von der Hand zu weisen. Damit verbunden wären aber nicht nur die bereits erwähnten Probleme im Bereich der touristischen Umsätze, sondern auch diverse ökologische Probleme (Massenandrang bei schönem Wetter, Verkehrsprobleme usw.).

Die schweizerische Tourismusbranche steckt in einem tiefgreifenden Wandel. Die Krise der letzten Jahre hat zahlreiche Schwächen aufgedeckt und zu einem rasanten Strukturwandel geführt. Diesem Strukturwandel werden verschiedene Anbieter zum Opfer fallen, und es ist zu hoffen, daß diejenigen, die überleben, gestärkt aus diesem Prozeß hervorgehen. Zusammen mit den neugeschaffenen Angeboten werden sie die Basis bilden, um den Herausforderungen der Zukunft zu begegnen. Ziel muß es sein, daß die Schweiz wieder vermehrt am internationalen Tourismus partizipieren kann.

\section{Literatur}

BALDENWEG-BÖLLE, U. '(1996): Reiseland Schweiz: Stärken, aber auch Schwächen. In: UBS (Hrsg.): Tourismus Schweiz. Lage und Perspektiven; Zürich.

BUNDESAMT FÜR STATISTIK (1996a): Der öffentliche Verkehr 1994. Statistische Resultate; Bern.

BUNDESAMT FÜR STATISTIK (1996b): Hotel- und Kurbetriebe in der Schweiz. Angebot und Nachfrage im Winterhalbjahr 1995/96; Bern.

BUNDESAMT FÜR STATISTIK (1997): Hotel- und Kurbetriebe in der Schweiz. Angebot und Nachfrage im Sommerhalbjahr 1996; Bern.

GENSLER, G. (1979): Wetter und Klima des Bündner Sommers. In: Terra Grischuna - Bündnerland. Zeitschrift für bündnerische Kultur, Wirtschaft und Verkehr, Nr. 3/79; S.138-141 HARTMANN, M. (1995): Blick in die Bündner Ferienwerkstatt. Bündner Beiträge zum Tourismusmanagement 2; Chur.

HARTMANN, M. (1997): Schweiz Tourismus - Der Wandel zum Marketing-Unternehmen im globalisierten Umfeld. In: KASPAR, C. (Hrsg.): Jahrbuch der Schweizerischen Tourismuswirtschaft 1996/97, S. 93-106; St. Gallen.

KASPAR, C./FREY, C. (1993): Tagestourismus - Ausmaß, Effekte und wirtschaftliche Bedeutung. Arbeitsunterlage zum Tourismus Nr. 4; St. Gallen.

KÜNZI, F./SCHMIDHAUSER, H.-P. (1989a): TOMAS, Touristisches Marktforschungssystem: Expertenbericht Sommerhalbjahr 1988; o. O.

KÜNZI, F./SCHMIDHAUSER, H.-P. (1989b): TOMAS, Touristisches Marktforschungssystem: Expertenbericht Winterhalbjahr 1988/89; o. O.

LORCH, J. (1995): Trendsportarten in den Alpen. Konflikte, rechtliche Reglementierungen, Lösungen; CIPRA (Hrsg.), Kleine Schriften 12/95; Vaduz.

MÜLLER, H.-R./KASPAR, C./SCHMIDHAUSER, H.-P. (1991): Tourismus 2001. Delphi-Umfrage 1991 zur Zukunft des Schweizer Tourismus; Bern.

NÖTHIGER, Ch. (1996): Sommertourismus in Graubünden. Entwicklung - Aktuelle Probleme - Zukunftschancen; Diplomarbeit am Geographischen Institut der Universität Zürich; Zürich.

NÜTZI, B. (1997): Ferienland Schweiz. Für Kurzurlauber attraktiv. In: Bulletin. Das Magazin der Credit Suisse, Nr. 3/97; S. $13-16$.

RÖTHLISBERGER, P. (Hrsg.) (1959): Bergbahnen der Schweiz. Eine allgemeinverständliche Darstellung der technischen und wirtschaftlichen Entwicklung der Zahnradbahnen, Standseilbahnen, Luftseilbahnen, Sessel- und Skilifte im klassischen Land der Bergbahnen; Siebnen.

SENGER, M. (1962): Zur Geschichte des Schweizerischen Fremdenverkehrs. Separatdruck aus Rätejahrbuch 1962, S. 161-205; Bern.

VERKEHRSVEREIN GRAUBÜNDEN (1994): Tourismus Plattform Graubünden (TP-GR); Chur.

ZEGG, R./MATTER, H.-J./INÄBNIT, M. (1993): Deshalb leben wir vom Tourismus...: Kurzfassung der Studie "Die wirtschaftliche Bedeutung des Tourismus in der Region Mittelbünden»; Chur. 